\title{
Evaluation of an Information Flow Gain Algorithm for Microsensor Information Flow in Limber Motor Rehabilitation
}

\author{
Naiqiao Ning and Yong Tang (iD \\ School of Sports, Hunan University of Humanities, Science and Technology, Loudi, Hunan 417000, China \\ Correspondence should be addressed to Yong Tang; 3070@huhst.edu.cn
}

Received 12 December 2020; Revised 26 February 2021; Accepted 13 March 2021; Published 22 March 2021

Academic Editor: Wei Wang

Copyright (C) 2021 Naiqiao Ning and Yong Tang. This is an open access article distributed under the Creative Commons Attribution License, which permits unrestricted use, distribution, and reproduction in any medium, provided the original work is properly cited.

\begin{abstract}
This paper conducts an evaluative study on the rehabilitation of limb motor function by using a microsensor information flow gain algorithm and investigates the surface electromyography (EMG) signals of the upper limb during rehabilitation training. The surface EMG signals contain a large amount of limb movement information. By analysing and processing the surface EMG signals, we can grasp the human muscle movement state and identify the human upper limb movement intention. The EMG signals were processed by the trap and filter combination denoising method and wavelet denoising method, respectively, the signal-to-noise ratio was used to evaluate the noise reduction effect, and finally, the wavelet denoising method with a better noise reduction effect was selected to process all the EMG signals. After the noise is removed, the signal is extracted in the time domain and frequency domain, and the root mean square (RMS), absolute mean, median frequency in the time domain, and average power frequency in the frequency domain are selected and input to the classifier for pattern recognition. The support vector machine is used to classify the myoelectric signals and optimize the parameters in the support vector machine using the grid search method and particle swarm optimization algorithm and classify the test samples using the trained support vector machine. Compared with the classification results of the grid search optimized support vector machine, the optimized vector machine has a $7 \%$ higher recognition rate, reaching $85 \%$. The action recognition classification method of myoelectric signals is combined with an upper limb rehabilitation training platform to verify the feasibility of using myoelectric signals for rehabilitation training. After the classifier recognizes the upper limb movements, the upper computer sends movement commands to the controller to make the rehabilitation platform move according to the recognition results, and finally, the movement execution accuracy of the rehabilitation platform reaches $80 \%$ on average.
\end{abstract}

\section{Introduction}

Traditional rehabilitation training is usually a long and repetitive process, mainly performed manually by a therapist or with the help of simple equipment to drive the affected limb, this training method is usually assisted by medical personnel, and the physical exertion of medical personnel in the rehabilitation process is very high, so it is difficult to ensure the intensity and durability of the rehabilitation training, limiting the optimization of the rehabilitation training and rehabilitation effect [1]. A physical rehabilitation robot is an automated device that assists or replaces a physician in completing rehabilitation training, using sensor feedback from human physiological data to perform rehabilitation training, which can compensate for the deficiencies of traditional rehabilitation training and enable more accurate and intelligent rehabilitation training [2]. The interaction between the robot and the patient can be divided into two categories, depending on the sensors used to obtain the movement intention during rehabilitation training: one is based on motion signals, such as position signals, force signals, and force-potential mixed signals, and the other is based on physiological signals, such as EMG signals and EEG signals [3]. The surface myoelectric signals have different degrees of correlation with muscle function and action patterns, are noninvasive, and can be measured and analysed in real-time, which has important practical value in clinical medicine and rehabilitation medicine [4]. Every action of the 
human body is realized by the central nervous system controlling muscle contraction, and in the process of muscle contraction, there will be myoelectric signals generated, which are bioelectric signals generated by muscle contraction in the process of action realization, and the action information contained in the myoelectric signals is ahead of the actual muscle action and can reflect the body's action intention earlier, so it can be used to predict body movements [5]. The sEMG can reflect the activity level of specific muscle groups, allowing for more detailed monitoring and control of limb movements [6]. It can also be collected by surface electrodes, eliminating the need for complex mechanical structures and avoiding the pain and cross-infection that can be caused by needle electrodes piercing the muscle [7]. It has been medically proven that active, conscious rehabilitation is more beneficial to patients than passive rehabilitation. In traditional rehabilitation training, patients just follow the training mechanically and repeatedly, which is boring and affects the training effect [8]. To create an engaging training environment for patients, we can use the surface EMG signals of the body as the control signal for the rehabilitation system to assist patients [9].

Jamwal et al. extended the single-channel myoelectric signal AR model with a multichannel surface myoelectric signal AR model, using a Bayesian classifier to distinguish between six different actions [10]. Kim's team in the US and North-Western University's team used surface EMG signals generated by nerves in the patient's residual arm to achieve control of the artificial limb [11]. The absolute mean, slope rate of change, waveform length, and cross-zero rate were selected as eigenvalues to form a feature vector, and an unsupervised linear classifier was used to identify the movement patterns of the hand, wrist, and elbow joints of the upper limb [12]. Zhang et al. performed a discrete wavelet transformation of the EMG signals collected from eight channels of the hand, then used the sum of the absolute values of the wavelet coefficients for each layer of the decomposition as a feature vector, and used the support vector machine as a classifier to classify the six movements of the hand [13]. For the rehabilitation robot, five time-domain feature vectors, namely, slope sign-transformation rate, over-zero rate, absolute value slope average, absolute value average, and waveform length, together with the fourthorder augmented reality model coefficients, were used to form a feature vector that mixed multiple parameters [14]. Finally, a Bayesian classifier was used to identify and classify multiple movement models of the upper limb [15]. Kim and Jeon used the pattern recognition of electromyographic signals to control the movements of the single and combined fingers of the prosthetic hand in different postures [16]. Liu et al. studied the structure of the human body and human movement function and designed a humanoid torso robot connected in series by UPS parallel platform, which simulates human movement by using the good spatial kinematics of UPS parallel platform and has certain kinematic ability, flexibility, versatility, and operability [17]. As the research on the human torso movement is getting deeper, scholars at home and abroad have also done a lot of research on the waist booster [18]. Through modelling of the mechanism and software simulation of motion analysis, experimental studies have shown that increasing the stiffness of elastic elements within a certain degree of lumbar flexion can help the human body perform bending exercises and protect the human lumbar spine [19].

In this paper, a surface electromyography (EMG) signal is collected from the human upper limb during rehabilitation training, and the EMG signal is analysed and processed by a classifier to identify the human movement intention. According to the essence of signal denoising, two denoising schemes are selected to denoise the signal, and the signal-tonoise gain is used to determine the final denoising scheme. After denoising the EMG signal, we extracted features in the time domain and the frequency domain, analysed the eigenvalues, and finally composed a mixed eigenvector from the root mean square (RMS) and absolute mean values in the time domain and the median frequency and mean power frequency in the frequency domain. The raw surface electromyography signals collected from the waist are processed for window segmentation and active segment detection, the processed signals are extracted with valid features, and the feature vectors are input to the classifier to train and recognize the motion. The extracted eigenvalues are used to train the support vector machine model, optimize the parameters using the grid search method and particle swarm algorithm, and finally verify the recognition rate using the test data set to select the optimization method with a good classification effect. The recognition and classification algorithm for EMG signals was applied to an upper limb rehabilitation training platform for experimental validation. Assuming that the left upper limb is the healthy side and the right upper limb is the affected side, we verify the feasibility of using myoelectric signals from the healthy side to drive the affected limb on the upper limb rehabilitation training platform.

\section{Microsensor Information Gain Algorithm Analysis and Design in Motion}

2.1. Sensor Information Gain Algorithm Design. The human motor system consists of the nervous system and skeletal muscles. Motor neurons transmit the movement intentions of the cerebral cortex to the skeletal muscles, thereby controlling muscle contraction and guiding the body's joints to move. When the muscles of the body contract, motor neurons produce a potential change in the muscle fibres, which is related to the distribution of positive and negative ions inside and outside the cells. Motor neurons transmit the excitations generated by the cerebral cortex to the muscle fibres, increasing the permeability of the muscle fibre cell membrane and allowing positive ions to enter the cell, so that the potentials inside and outside the membrane are reversed compared to the resting state, changing from positive external and internal to negative external and internal [20]. A potential difference is generated between the contracted and uncontracted muscle fibres, resulting in the formation of an action potential that returns to the resting potential at the end of the exercise, as shown in Figure 1. The surface myoelectric signal is the muscle electrical signal 


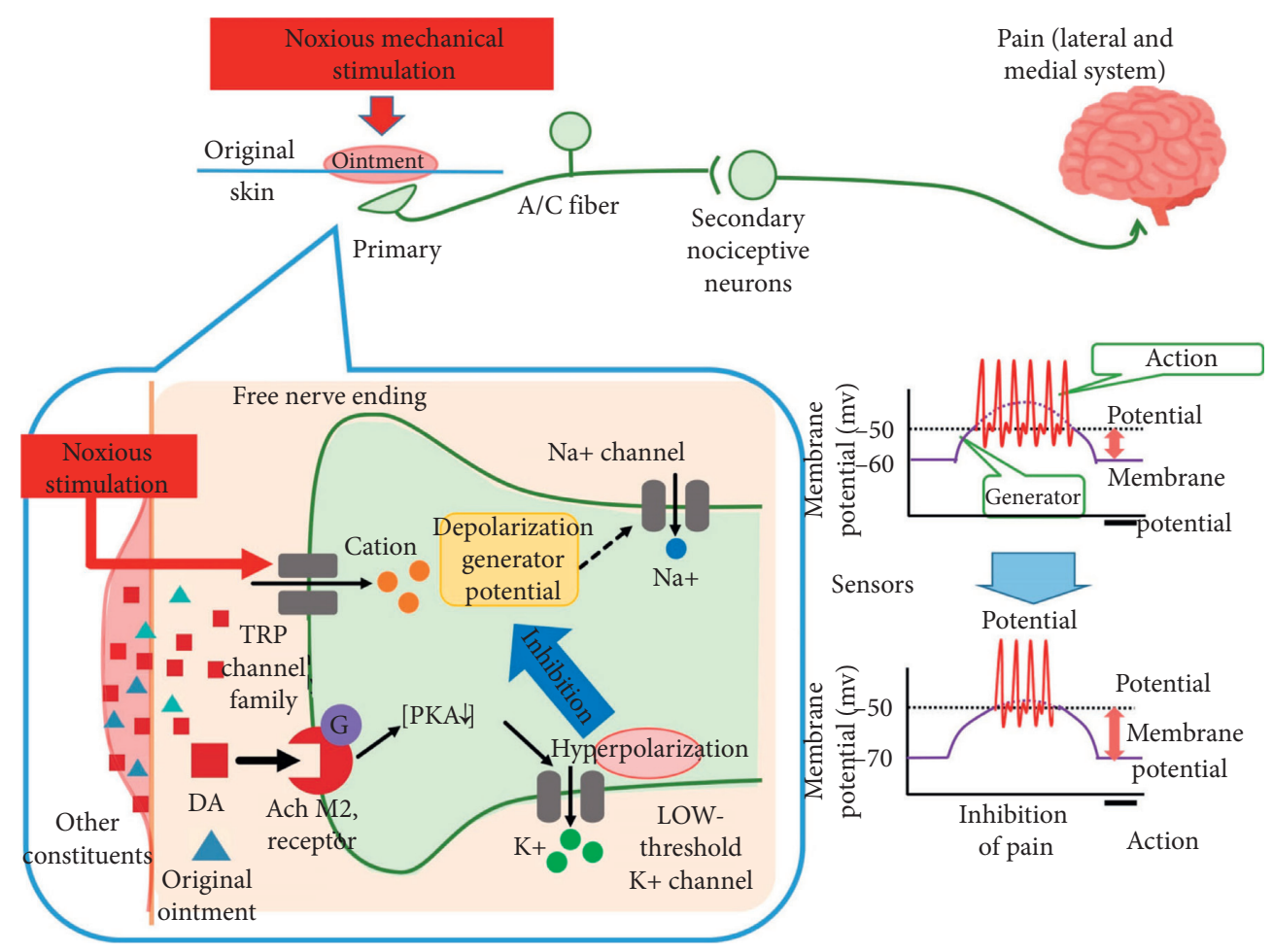

FIgURE 1: Mechanism of surface EMG signal generation.

obtained on the surface of human skin through surface electrodes during muscle contraction.

When acquiring EMG signals, to maximize the signal and minimize electrical interference from neighbouring muscles to the target muscle, the silicon rod should be placed perpendicular to the direction of the muscle fibre and in the central area of the muscle to be tested, away from the tendon and the edge of the muscle. The sensor is marked with arrows on its surface to help determine the correct orientation, and the Trigon system provides a dedicated adhesive interface to simplify sensor attachment. The use of this interface effectively facilitates a high-quality electrical connection between the sensor's silicon rod and the sensor.

The EMG data collected by the DELAYS wireless surface electromyography system will be converted to CSV using the Delays File Conversion Utility, which comes with the system's format conversion unit, to facilitate subsequent processing of the data using MATLAB. Before the EMG signal can be acquired, a new tester profile must be created to record information about the person and the different parts of the muscle to be acquired, including the subject's name, age, height, weight, and the location where the signal was acquired [21]. After the basic information is filled in, since not all sensors are used in motion acquisition, you need to select the sensor, that is, the acquisition channel. Once the acquisition channel is identified, you will enter the acquisition interface, where you can perform data acquisition and display the corresponding motion waveform according to the set motion mode. Surface EMG is a signal of nerve activity guided and recorded from the skin surface by the application of surface electrodes. It is the combined effect of the electrical activity signal of the nerve stem and the myoelectric signal of the superficial muscle in time and space and is related to the state and function of the muscle activity, the position of the surface electrode, the thickness of the subcutaneous fat, the individual's body temperature, and other factors.

Thus, EMG signals can reflect the state of neuromuscular activity. They are of great value in clinical medicine, sports medicine, biomedicine, and engineering. Surface EMG signals record the integrated activity of the whole muscle and all motor units within a muscle group during muscle movement. The EMG signals of different individuals vary greatly from one individual to another, and the EMG signals extracted from different muscle parts of the same body may also be different but still have a certain regularity: when the human body performs different body movements, the contraction patterns of the muscles are different, and the differences between the different patterns can be characterized by the characteristic differences in the surface EMG signals; thus, there is a correlation between the surface EMG signals and the human body movements [22]. When muscle fatigue is not considered, if the same muscle is selected and the same movement is repeated several times, the EMG signal collected will not change much, so the EMG signal has good repeatability. Therefore, the specific amount of EMG signal can be used to identify different actions of the limb, and the EMG signal can be used as stable and reliable control information for the upper limb rehabilitation robot, as shown in Figure 2.

The DELAYS wireless surface EMG acquisition system consists of a base station that receives sensor information and 16 EMG sensor nodes, as shown in Figure 2. The system is capable of simultaneous detection of 16 channels, and each 


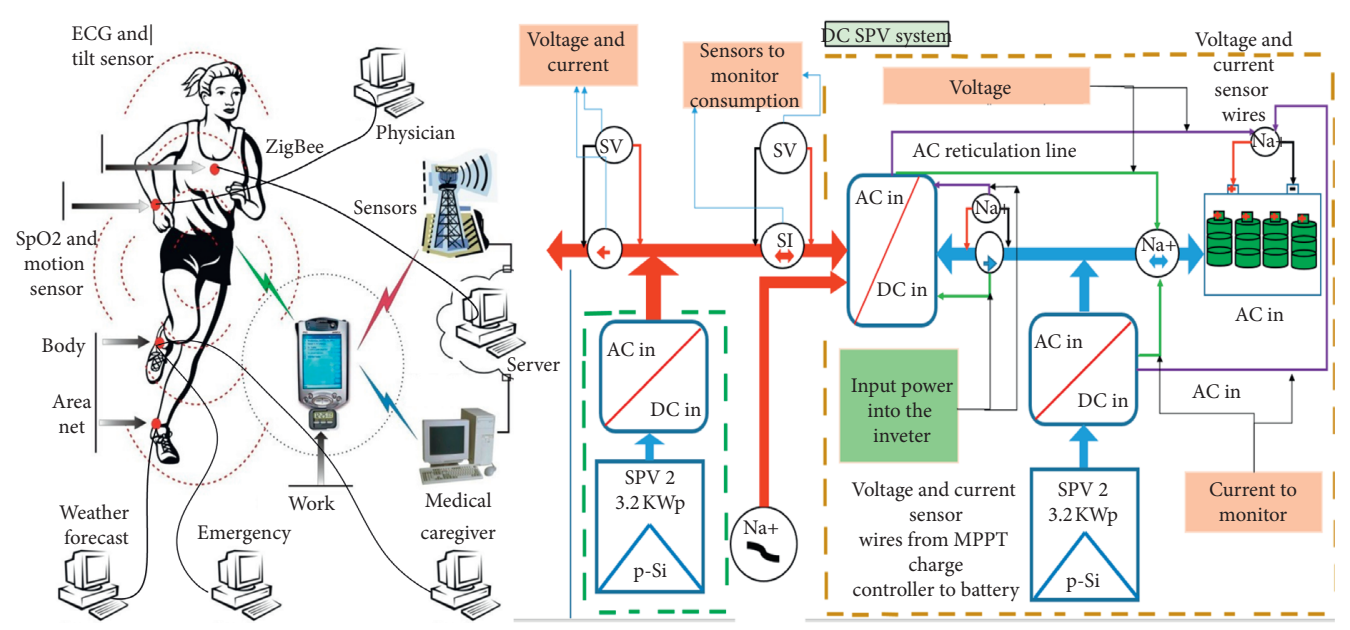

FIGURE 2: Sensor information gain algorithm model.

channel is capable of independent signal transmission. The EMG measurement device in the system is a Trigon wireless sensor, shown in Figure 2, which uses four silicon rods in contact with the skin to collect EMG signals from the human skin surface. The sensor has a fixed distance of $10 \mathrm{~mm} * 10 \mathrm{~mm}$ between the positive and negative electrodes to ensure repeatability and accuracy of the data for each test. The sensor uses double electrodes stacked on top of each other to ensure signal reliability [23]. The wireless acquisition mode of the sensor realizes wireless data transmission through a wireless interface. The reliable working distance is $20 \mathrm{~m}$, and the sampling frequency is $2000 \mathrm{~Hz}$. When using the system to collect the subject's EMG signal, the sensor will wirelessly transmit the collected realtime EMG signal to the base station, and the base station will transmit the received information to the computer through the computer's USB interface. The data reading, storage, and subsequent processing of the electrical signals are shown in Figure 2.

According to the characteristics of the surface EMG signal, the frequency range of the EMG signal is $0-500 \mathrm{~Hz}$; because of the influence of the EMG signal acquisition equipment, the original EMG signal will be mixed with a lot of noise, so it is necessary to filter the original signal to obtain useful information of the EMG signal. In this paper, a low-pass filter is used to remove the unwanted high-frequency signal from the original EMG signal; then, a trap filter is used to remove the IFE interference from the original signal, and an AIR zero-phase shift digital filter is used to correct the baseline drift. In this paper, a classical Butterworth filter is used to design a secondorder Butterworth filter for low-pass filtering of the myoelectric signal, the transfer function of which is given by equation (1):

$$
H\left(e^{j w}\right)=\frac{b(1)+b(2) e^{-j w}-b(3) e^{-2 j w}+\cdots+b(7) e^{-6 j w}-b(8) e^{-7 j w}}{a(1)+a(2) e^{-j w}-a(3) e^{-2 j w}+\cdots+a(7) e^{-6 j w}-a(8) e^{-7 j w}} .
$$

The comparison between the original EMG signal and the original EMG signal after the signal is processed by the trap is shown in equation (2). The frequency characteristics after the trap treatment are rapidly depressed at $50 \mathrm{~Hz}$, and the rest of the frequency band is normal, so the $50 \mathrm{~Hz}$ IF interference in the EMG signal can be effectively removed.

$$
H\left(e^{j w}\right)=\frac{1+\alpha e^{-j w}+\beta e^{-j w}-\chi e^{-j w}}{1-\alpha e^{-j w}-\beta e^{-j w}+\chi e^{-j w}} .
$$

Wavelet transform is a method of converting a onedimensional signal in the time domain to a two-dimensional signal in the time-frequency domain and then analysing the nonstationary signal in the time-frequency domain, with multiresolution analysis characteristics [24]. The basic idea of the wavelet transform is to translate a wavelet basis function through the translation and scaling method and to obtain the following from the product of the original signal at different scales:

$$
W_{c}(a, b)=\int_{-\infty}^{+\infty} \varphi_{a, b}(t) c_{a, b}(t) \mathrm{d} t
$$

where the wavelet basis function in the wavelet transform is a bandpass filter whose Fourier transform must satisfy condition (4):

$$
W_{c}=\int_{R} \frac{\varphi^{2}(w)}{w} \mathrm{~d} w
$$

The wavelet fundamental function is called the wavelet function after stretching a and translating $b$. The wavelet sequence constitutes a set of orthogonal bases of the squared product space.

$$
\varphi_{a, b}(t)=\frac{2}{\sqrt{a_{i}^{j}}} \varphi^{2}\left(\frac{t-b_{i} a_{i}^{j}}{b_{i}^{j}}\right)
$$

The above is about the continuous wavelet transform, but the discrete wavelet transform is mainly used in signal processing, usually by using the Mallet algorithm, the main idea of which is to perform the wavelet transform on the larger signals first and then do the wavelet transform again on the $1 / 2$ scale of the original scale for the high-frequency signals. To obtain the discrete wavelet transform, the scale factor a and the translation factor $b$ are first discretized; that is, 


$$
\varphi(t)=\left\{\begin{array}{l}
a_{i}^{j}, \\
k b_{i} a_{i}^{j} .
\end{array}\right.
$$

Then, the discrete wavelet function is

$$
\varphi_{a, b}(t)=\frac{2}{\sqrt{a_{i}^{j}}} \varphi\left(\frac{t-k b_{i} a_{i}^{j}}{b_{i}^{j}}\right) .
$$

Discrete wavelet transforms to

$$
W_{i, j}(t)=\int_{-\infty}^{\infty} c(t) \varphi_{a, b}(t) \mathrm{d} t .
$$

Based on familiarity with the wavelet transform, the principle of wavelet denoising is obtained in conjunction with the purpose of denoising the original electromyographic signal. The useful signals in the EMG signal are usually the low-frequency signals in the original EMG signal or those that are relatively smooth and do not fluctuate much, while the noisy signals are some high-frequency signals. When performing signal processing, we describe the acquired surface EMG signal as a one-dimensional signal model.

$$
c(i)=s(i)+\delta \times e(i)-\delta^{e(i)} .
$$

The basic idea of wavelet denoising based on wavelet transform is to decompose the mixed noise signal, then remove the wavelet component of the high-frequency part of each decomposition scale, while the remaining wavelet spectrum is the wavelet spectrum of the original signal, and carry out wavelet inversion to construct the original signal, to obtain the signal after denoising.

When the number of decomposition layers is too many, the resulting signal will drown out the information in some frequency bands because the decomposition is too dense; if the number of decomposition layers is too small, the noise cannot be removed better, so the number of decomposition layers cannot be too big or too small. However, there is no fixed empirical formula for choosing the number of decomposition layers, so we need to repeatedly test and verify according to the data, signal characteristics, and experimental results. When applying wavelets to denoise the EMG signal, the signal is decomposed into two to five layers. When the base function and threshold selection algorithm are the same, only the number of decomposition layers is changed, and the signal denoising of four channels during elbow flexion in subject 1's first group is compared in many experiments. The best denoising effect is obtained when the signal is decomposed into three layers, and the three-layer decomposition is finally chosen for denoising.

$$
D_{i}(k)= \begin{cases}\operatorname{sign}\left(D_{i}(k) *\left(D_{i}^{2}(k)+\alpha D_{i}(k)\right)\right), & D_{i}(k) \leq \lambda, \\ 1, & D_{i}(k)>\lambda .\end{cases}
$$

2.2. Microsensor Information Flow Analysis Design. The lower limb gait analysis system mainly consists of inertial sensors, a data forwarding router, a computer terminal, and the composition of the form called body sensor network and specifically refers to human-centered, integrated intelligent sensors, biomedical, mechatronics, data acquisition and preprocessing, multisensor data fusion, artificial intelligence, wireless communication, and other pieces of multidisciplinary knowledge, to achieve the collection, fusion, and calculation of the human body's various movement data. The 7 inertial sensors in the lower limbs of the human body are used as the data acquisition layer of the whole system, and these 7 sensors are bound to the back of the left foot, back of the right foot, left calf, right calf, left thigh, right thigh, and hip of the human body, to collect the acceleration, angular velocity, and magnetic field value of each part of the magnetic field, as shown in Figure 3. The transmission rate of the inertial sensor is $60 \mathrm{~Hz}$, the indoor transmission distance is $40 \mathrm{~m}$, the outdoor transmission distance is $110 \mathrm{~m}$, and the working time is $10 \mathrm{~h}$. The network forwarding layer is the data forwarding router, and the data of the inertial sensor are forwarded to the computer terminal by the router in the form of $2.4 \mathrm{G}$ WIFI forwarding. The router of the data forwarding layer is shown in Figure 3. The data forwarding router collects data from multiple inertial sensors and connects them to the computer terminal via USB cable. In this paper, the data fusion of inertial sensors, lower limb attitude fitting, the calculation of gait parameters, and simulation of various algorithms are completed on the computing terminal.

To improve the measurement accuracy of inertial sensors, data preprocessing methods for gyroscopes, accelerometers, and magnetometers are studied. Commonly used data preprocessing methods are low-pass filtering, high-pass filtering, data smoothing filtering, wavelet changes, and so on. In the following, the inertial sensor data are preprocessed through comparative experiments. As the inertial sensor system has been for the internal gyroscope and accelerometer output results of high-pass filtering and low-pass filtering, so this section only does the data smoothing preprocessing. Data preprocessing to meet the prerequisites for the preprocessing calculations as simple as possible cannot produce a large delay, because the design of the first principle of the lower limb gait analysis system is real-time, if the data preprocessing is too complicated, it will lead to a relatively long calculation time, and the data will have a lag There are several common data smoothing filters, such as median filtering, judgment filtering, mean filtering, and weighted mean filtering. Among them, the median filter is the filter with a smaller computation amount and simpler operation; therefore, this paper adopts the median filter for acceleration and angular velocity data preprocessing; the preprocessing flow is described as follows: due to different magnetic field interference in different environments, the data processing of magnetometers is relatively complex [25].

In this paper, the output data of the magnetometer is processed by the threshold method. The key of the threshold method is the selection of the threshold value, which will have a great influence on the yaw angle calculation if the threshold value is too large or too small. This method is too ideal because the actual magnetic field of the environment is not equal to the local magnetic field. After all, the environment is usually in the corridor, laboratory, ward, and 


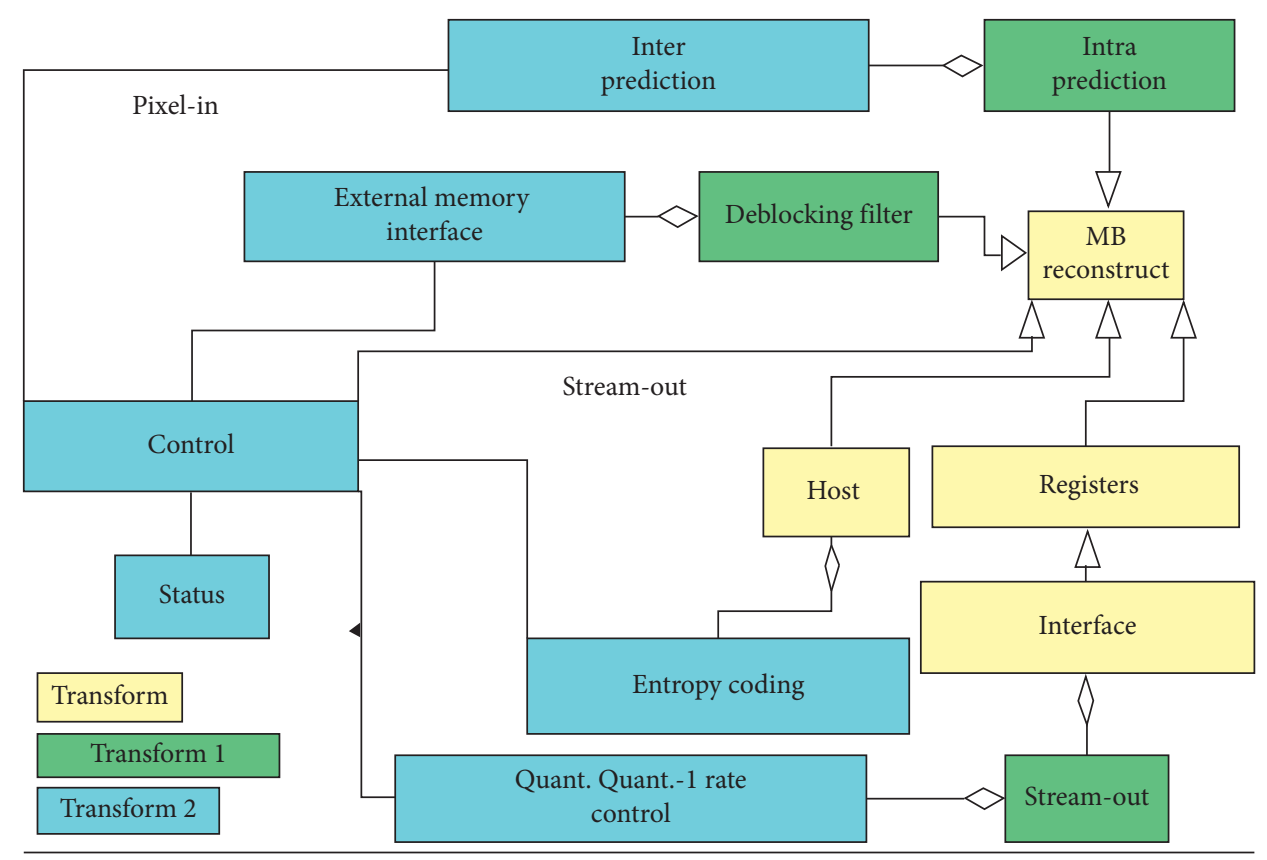

Figure 3: Microsensor information flow design.

other places; there must be all kinds of electrical appliances and communication cables in the above places. For example, there are steel bars in the floor slab, which will affect the Earth's magnetic field. In this paper, the magnetic field after ellipsoidal fitting is chosen as the threshold value. The advantage of this approach is that, in the ellipsoidal fitting process, the sensor is generally rotated in the air at a height greater than $30 \mathrm{~cm}$ from the ground, and the magnetic field interference generated by the sensor circuit board is compensated during the ellipsoidal fitting process. In summary, it is a good choice to select the magnetic field after ellipsoidal fitting as the threshold value. The purpose of the threshold method is to filter out the strong magnetic interference.

First, find the average value of the surface EMG signal, and then find the norm of the average value. Use the window function to perform moving average processing on the instantaneous energy of the surface EMG signal. The obtained value is judged with the appropriate threshold value, and the signal is greater than the threshold value. It is the action potential segment, and the value less than the threshold is the resting potential segment.

The errors of the magnetometers are mainly in three aspects: one is the process error produced by the manufacturer of the magnetometers, such as the materials of the magnetometer, the production process, and the advanced degree of the production line; the second is the assembly and installation error, such as whether or not the module manufacturers compensate the magnetometers for the threeaxis orthogonality error; the third is the influence of the surrounding environment on the magnetometers, such as the indoor and outdoor floor steel and the nearby cell phones. The magnetic field generated by ferrous materials such as magnetic tape and watches can affect the accuracy of the magnetometer. Add speech recognition module and speech generation module, the speech recognition module adopts LD3320 board, and LD3320 is a speech recognition board based on nonspecific speech recognition (SI-ASR: speaker-independent Automatic Speech Recognition) technology and keyword list technology. The list of keywords can be set according to the application requirements, as shown in Table 1. The recognizable commands are as follows: prepare to recognize, start to recognize, stop collection, pull line data, Euler angle data, display curve, continuous curve, stop curve, and return. The above voice commands can meet the data acquisition and display requirements of the rehabilitation robot.

The main interface mainly includes a dynamic display of voice information, sensor data display, and function buttons. The dynamic display of voice information shows the current speech recognition detection status and recognition results, which is convenient for the user to understand the current state of the speech system. The middle part displays the voice password, which is the primary password and secondary command for speech recognition. On the right side are functional keys, which users can touch to control according to their needs.

Due to the nonlinear and unstable nature of EMG signals, signal processing and analysis are required. In this chapter, myoelectric signal pattern recognition mainly includes the acquisition, signal preprocessing, feature extraction, and signal classification of the lumbar myoelectric signal, as shown in Figure 4. First, preprocessing of the collected EMG signal from the lumbar region of the subject is performed, and the sliding window is used to segment the signal and extract the active segment data. Feature extraction and pattern classification are the two core tasks of pattern recognition system design. It is necessary to extract the multidimensional vectors that can represent the motion 
TABLE 1: Speech recognition commands.

\begin{tabular}{lccc}
\hline Identifier & Coding & String & Values \\
\hline CODE_KL & 1 & Reasonable text font size & 4 \\
CODE_TZCJ & 2 & Icon metamorphosis & 8 \\
CODE_LXSJ & 4 & Interactive feedback & 3 \\
CODE_OLJS & 57 & For people with weak hearing & 58 \\
CODE_XSQX & 58 & Expand the area of the interactive area & 47 \\
CODE_JXQX & 75 & Improve the ease of operation & 25 \\
CODE_ZTQX & 85 & Sight or touch can be used to compensate & 14 \\
CODE_FH & 14 & Multisensory channels & 27 \\
\hline
\end{tabular}

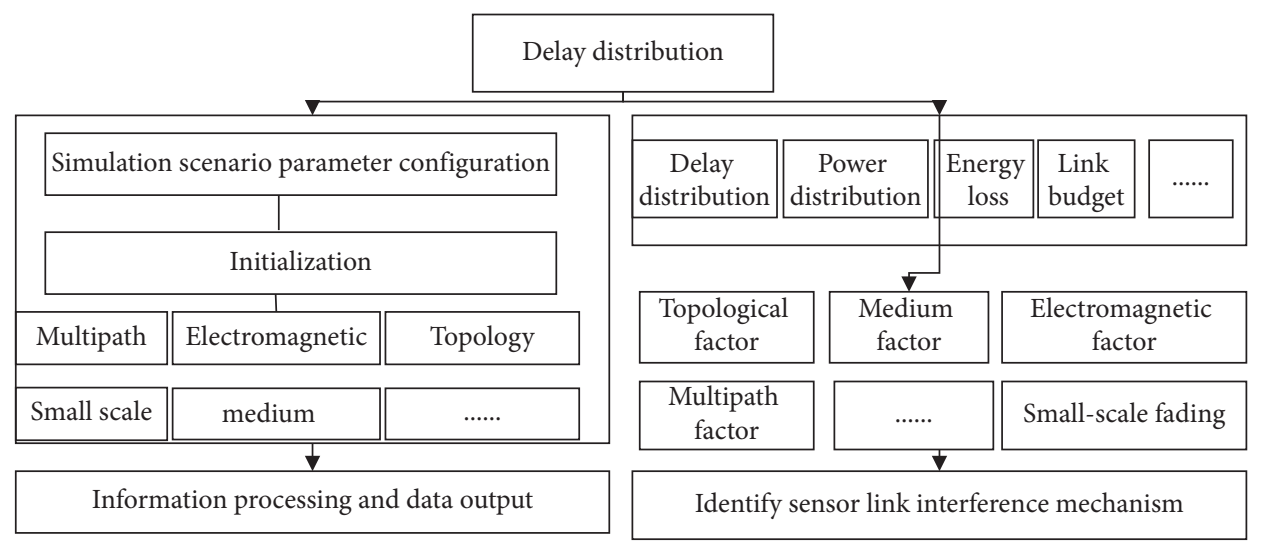

FIGURE 4: Flowchart of myoelectric signal classification.

characteristics hidden in the original signal. Commonly used feature extraction methods are time-domain feature extraction, frequency domain feature extraction, and timefrequency domain feature extraction. The extracted feature matrix is used as the input matrix for pattern recognition, and different feature extraction methods have a great influence on the subsequent motion pattern recognition. Different classification algorithms have different classification results for the same feature vector, and a reasonable choice of classification algorithm determines the pattern recognition effect. Therefore, this chapter selects the optimal pattern recognition algorithm for lumbar EMG signals through analysis and comparison.

As the sEMG signal is continuous, it is often necessary to segment the obtained raw data to extract the characteristic information in the original signal and facilitate the analysis of the relationship between the signal and the action. Considering the characteristics of the EMG signal and the effective duration of the experimental acquisition, the data segmentation of the lumbar EMG signal was performed using the overlapping segmentation method.

As for the EMG signal on the surface of the rectus femoris muscle, the muscle is far away from the heart and is subject to little interference from the ECG signal, and the noise sources are mainly high-frequency ambient and radiation noise and and low-frequency motion artifacts and baseline drift noise. Currently, widely used digital filtering algorithms are mainly arithmetic mean filtering, median filtering, and so on; these filtering algorithms are simple to calculate, can overcome the interference of large pulses in the signal, and suppress small-amplitude high-frequency noise but cannot effectively eliminate the frequency domain noise of surface EMG signals in the high-frequency band and low-frequency band; time-frequency domain denoising methods mainly include wavelet denoising, empirical modal decomposition denoising, and these methods. It is capable of describing the local information of the signal, separating the high-frequency and low-frequency parts, with multiscale analysis, multichannel filtering, and ideal filtering effect, but it needs to decompose and reconstruct the signal several times by scaling factor and translation factor, which is computationally intensive and affects the real-time performance of the system.

\section{Results Analysis}

3.1. Identifying Result Analysis. In this paper, a total of 250 groups of experimental data were obtained, including 50 groups each for flat walking, stair climbing, stair descending, and uphill and downhill walking, and each group was preprocessed to detect the action start point and extract eigenvalues from the $200 \mathrm{~ms}$ data after the start point. Since the number of steps and the frequency of steps were different in each group, the number of gait samples extracted was also more or less. The number of gait samples from 250 groups of experimental data was counted, resulting in a total of 262 flat ground walking samples, 206 stair-up samples, 299 uphill samples, 203 downhill samples, and 354 downhill samples. Five different sample data were randomly selected $70 \%$ as a training set and $30 \%$ as a test set to validate the model. According to the importance score of the features, the features are added gradually as the input training model of 
the XGBoost algorithm, and the recognition accuracy is calculated on the test set. When the number of eigenvalues is 8 , the recognition rate reaches the highest, $87.69 \%$, and then the recognition rate starts to stabilize by adding more eigenvalues, which has very little influence on the recognition result. The result data in Figure 5 come from 10 results we randomly selected from 250 data.

To verify the validity of XGBoost for recognizing gait patterns, a linear classifier support vector machine (SVM), a nonlinear classifier back propagation neural network (BPNN), and a linear classifier support vector machine were selected as inputs. Random forest (RF) of the Bagging strategy is used to calculate the recognition rate of different classification algorithms on the test set, and the recognition rate is shown in Figure 5. XGBoost has the highest recognition rate. From the feature space distribution shown in Figure 6, we can see that the spatial distributions of the same eigenvalues in different gait often overlap with each other, and it is not easy to find a clear boundary for classification in the same area of the feature space. XGBoost does not try to find the correct classification boundary directly, but rather by learning from mistakes, introducing a weak classifier in each iteration to overcome the shortcomings of the existing combination of weak classifiers. The classifiers integrated into a strong classifier for accurate identification of different gait states, and the XGBoost leaf node values are calculated based on the samples, not simply averaged or median values, for a better fit to the sample details.

Surface electromechanical delay (EMD) signals are generally $10 \mathrm{~ms}$ to $100 \mathrm{~ms}$ ahead of the action, while joint angle signals and muscle force signals are also acquired by sensor detection, which may also have a delay problem. As shown in Figure 7, asynchronous analysis of the normalized EMD and joint angle signals shows that there is an obvious EMD between them. Therefore, to build a more accurate model for muscle strength prediction, the signals need to be synchronized to reduce the delay between them. Analysis of the correspondence between EMD signals and joint angle and muscle force shows that the EMD signals generally precede the joint angle and muscle force signals. As shown in Figure 7, this paper corrects the EMD by analysing the delay interval between signals adjacent to the peak.

Since muscle contraction produces both EMG and force and there is a nonlinear correlation between them, EMF values are difficult to measure or calculate directly because they are influenced by muscle fibre length and muscle fatigue. Also, since muscle force is influenced by muscle fibre length and muscle fatigue, it is difficult to measure or calculate muscle force directly, so it can be predicted and estimated by analysing the relationship between muscle force and myoelectricity. Currently, the study of muscle strength prediction can be divided into two strategies: the model method and the "black box" method. The model method requires the study of the underlying mechanisms of human body movement, analysing the relationship between bones, muscles, nerves, and so on, and then building similar bionic models to simulate body movement and estimate the resulting muscle force, such as the representative Hill musculoskeletal model; the "black box" method is

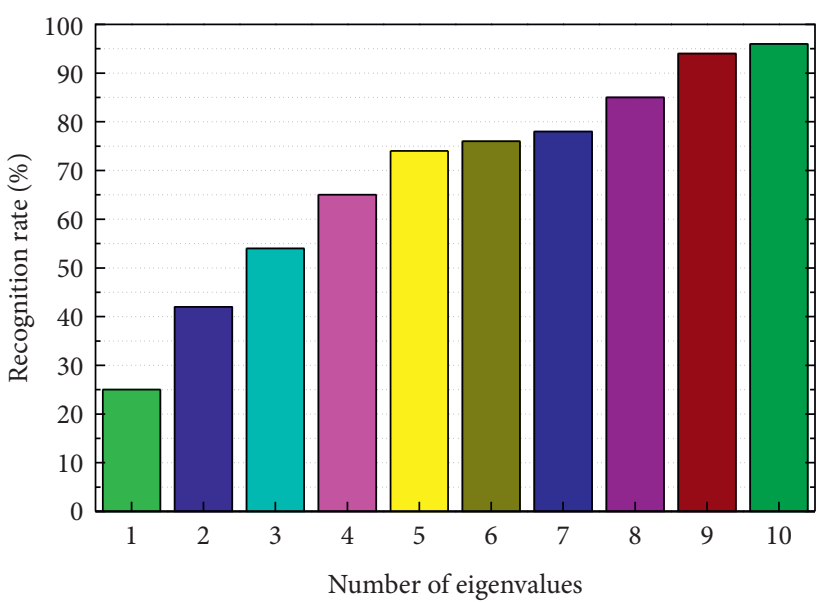

FIGURE 5: Relationship between the number of features and the recognition rate.

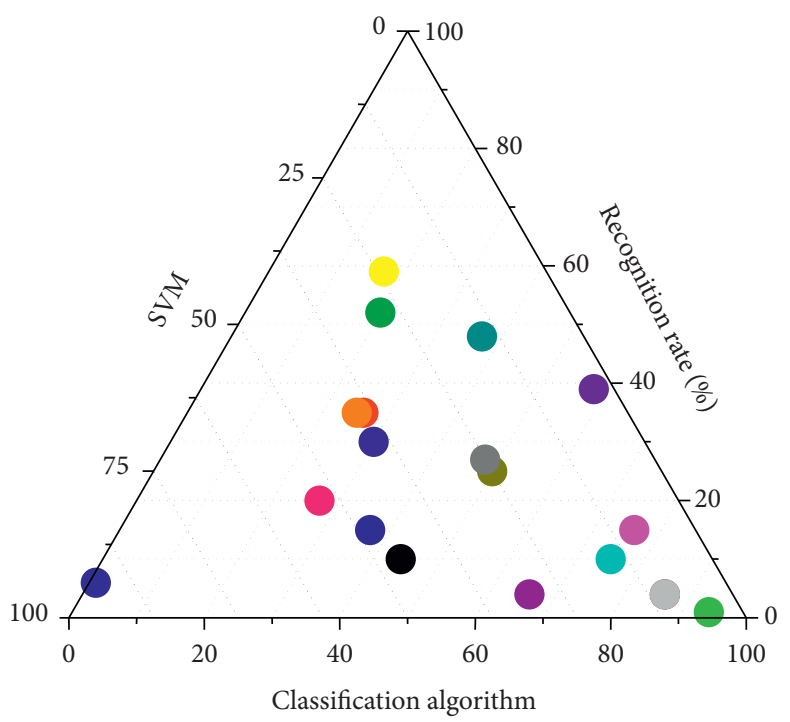

Figure 6: Comparison of identification results of different classification algorithms.

physiological and mathematical-statistical. A model of the relationship between muscle strength and the signals of the relevant features does not require the study of specific interconnections between them or require a complex modelling process, and different models for different people have better adaptability and applications, such as estimating muscle strength using neural networks. This is an analysis study of electromyography and muscle strength.

3.2. Experimental Results and Analysis. All the data in this paper were obtained by analysing and processing the surface EMG signals of the upper limbs of 10 subjects, each of whom performed four movements, elbow flexion, elbow extension, shoulder abduction, and shoulder dorsiflexion, and each of whom repeated each movement for five sets. The RMS, mean absolute value, median frequency, and mean power frequency of each action are used to form a feature vector to characterize the EMG signal category, so that each action has 


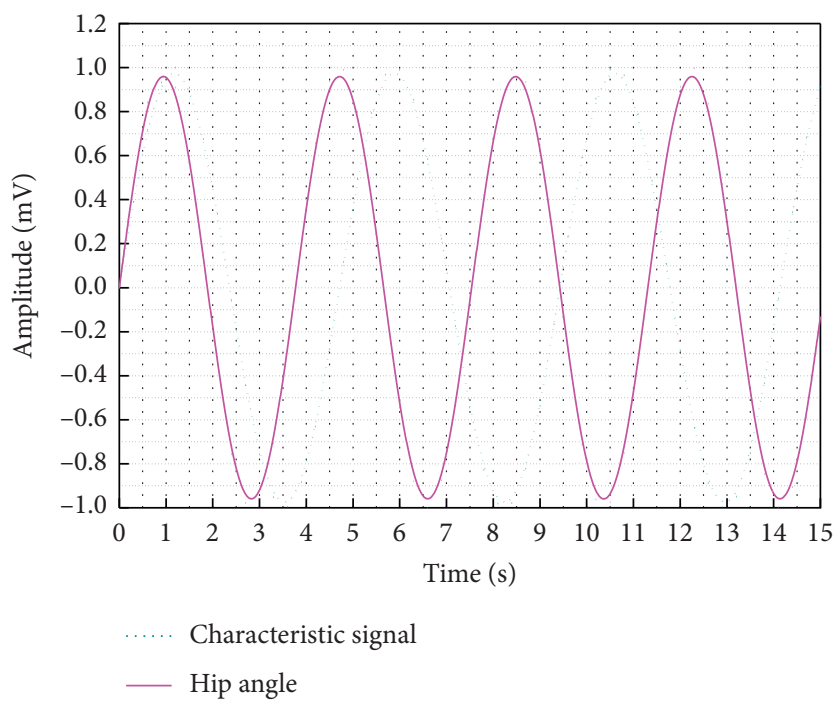

Figure 7: EMG signatures and hip angle.

50 sets of data, and each set consists of 16 feature elements. The training set was used to train and generate the classification model, while the test set was used to test the classification model and identify whether it could be classified or not. To identify and classify the four types of upper limb elbow flexion, elbow extension, shoulder abduction, and shoulder dorsiflexion, it is necessary to define a label for each of the four movements.

When identifying an action, an action corresponds to a label, and the classification result can be judged by the label definition. There are two types of incorrect classification: first, the classification result does not match the actual type, and it shows other action types; and second, the action attribution is not recognized.

The data were fed into the grid search optimized vector machine classifier and the PSO-optimized classifier for recognition, and the classification results are shown in Figure 8. From the two tables above, the recognition rate for a single action optimized by grid search ranged from $77.45 \%$ to $82.74 \%$, while the recognition rate for four actions per experimenter ranged from $75.35 \%$ to $83.25 \%$. The PSO-optimized classification method has significantly improved the pattern recognition accuracy compared to the nonoptimized one, with the recognition rate of individual actions ranging from $82.98 \%$ to $90.13 \%$ and the recognition rate of four actions per participant ranging from $84 \%$ to $87 \%$. The particle swarm is efficient in finding the penalty and kernel parameters in reducing the recognition error of the vector machine and improving the correct pattern recognition rate.

If the movements performed by the subject's right upper extremity are the same as those performed by the left upper extremity, the movements are correct; if the movements performed by the subject's right upper extremity are not the same as those performed by the healthy left upper extremity, the movements are incorrectly performed. During the experiment, the platform will search for the smallest trajectory $r$ in the database

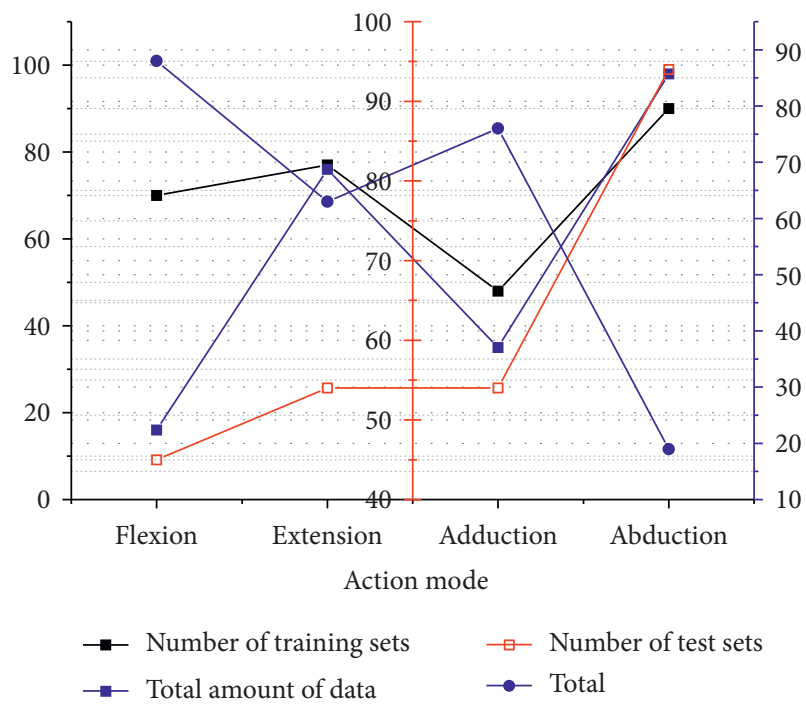

FIGURE 8: Data set classification.

according to the action categories outputted by the classifier. Because elbow flexion and extension and shoulder abduction and adduction are a pair of symmetrical movements, they were verified in pairs during the specific exercise process, and the trajectory of subject 1's 20 exercises is shown in Figure 9. During the experiment, each participant's movements were recorded, and the results of the upper extremity rehabilitation training platform for 10 subjects are shown in Figure 10.

From Figure 10, we can see that the average accuracy of the ten subjects' movement execution experiments on the rehabilitation training platform reached $80 \%$, which is not high enough but also confirms the feasibility of using the myoelectric signals from the healthy side to drive the movement of the affected limb on the experimental rehabilitation platform so that the patients can drive the affected upper limb according to their exercise will. 


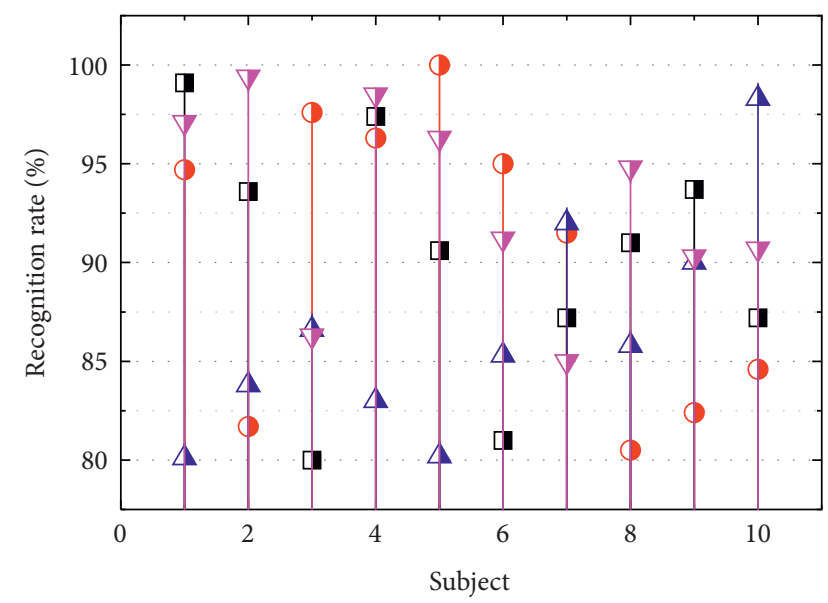

$\begin{array}{ll}\text { - Flexion } & \boldsymbol{\Delta} \text { Adduction } \\ \text { - Extension } & \nabla \text { Abduction }\end{array}$

Figure 9: Classification results.

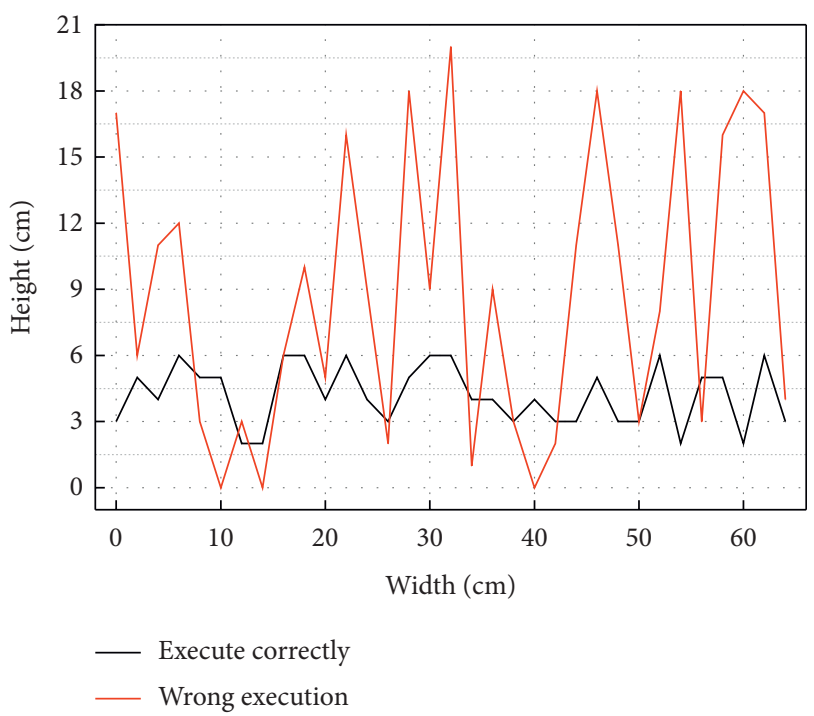

FIgURE 10: Experimental motion trajectory diagram.

\section{Conclusion}

According to the characteristics of the human waste movement, the structure of the robot is designed by combining the advantages of large space and flexible control of the flexor cable, which mainly includes three parts: the lower limb auxiliary exoskeleton, the upper body fixation mechanism, and the flexor-driven platform; kinematic modelling analysis is performed on the designed mechanical structure, and the relationship between the waist angle and the length change of the four flexor cables can be obtained. The driving force required for moving each flexor cable can lay a theoretical foundation for controlling the motor later. To ensure the safety of the patient, the sensor signals are fused. The motion of the motor-driven platform is controlled by a multisensor differential fusion PID algorithm, and the error value is within the controllable range when comparing the collected cable displacement sensor data with the theoretical simulation data. To obtain a better recognition rate, we optimize the parameters by using the grid search method and particle swarm algorithm, input the sample set into the vector machine model for training to obtain the final classification model, and validate it by using the test set, which results in an average motion recognition rate of $85 \%$, $7 \%$ higher than the $78 \%$ optimized by the grid search method.

\section{Data Availability}

The data used to support the findings of this study are available from the corresponding author upon request.

\section{Conflicts of Interest}

The authors declare that they have no known competing financial interests or personal relationships that could have appeared to influence the work reported in this paper.

\section{References}

[1] M. O. Al Kalaa and H. H. Refai, "Monitoring radiated coexistence testing using GMM-based classifier," IEEE Transactions on Vehicular Technology, vol. 66, no. 11, pp. 10336-10345, 2017.

[2] R. Punj and R. Kumar, "Technological aspects of WBANs for health monitoring: a comprehensive review," Wireless Networks, vol. 25, no. 3, pp. 1125-1157, 2019.

[3] M. S. Mahmud, H. Fang, and H. Wang, "An integrated wearable sensor for unobtrusive continuous measurement of autonomic nervous system," IEEE Internet of Things Journal, vol. 6, no. 1, pp. 1104-1113, 2018.

[4] J. Pagan, R. Fallahzadeh, and M. Pedram, "Toward ultra-lowpower remote health monitoring: an optimal and adaptive compressed sensing framework for activity recognition," IEEE Transactions on Mobile Computing, vol. 18, no. 3, pp. 658-673, 2018.

[5] P. O. McKeon and L. Donovan, "A perceptual framework for conservative treatment and rehabilitation of ankle sprains: an evidence-based paradigm shift," Journal of Athletic Training, vol. 54, no. 6, pp. 628-638, 2019.

[6] C.-Y. Ko, J. Ko, H. J. Kim, and D. Lim, "New wearable exoskeleton for gait rehabilitation assistance integrated with mobility system," International Journal of Precision Engineering and Manufacturing, vol. 17, no. 7, pp. 957-964, 2016.

[7] G. Chen, Z. Zhou, B. Vanderborght, N. Wang, and Q. Wang, "Proxy-based sliding mode control of a robotic ankle-foot system for post-stroke rehabilitation," Advanced Robotics, vol. 30, no. 15, pp. 992-1003, 2016.

[8] R. K. Elnaggar and M. F. Elbanna, "Evaluation of independent versus integrated effects of reciprocal electrical stimulation and botulinum toxin-A on dynamic limits of postural stability and ankle kinematics in spastic diplegia: a single-blinded randomized trial," European Journal of Physical and Rehabilitation Medicine, vol. 55, no. 2, pp. 241-249, 2019.

[9] S.-B. Ju and G. D. Park, "Effects of the application of ankle functional rehabilitation exercise on the ankle joint functional movement screen and isokinetic muscular function in patients with chronic ankle sprain," Journal of Physical Therapy Science, vol. 29, no. 2, pp. 278-281, 2017. 
[10] P. K. Jamwal, S. Hussain, N. Mir-Nasiri, M. H. Ghayesh, and S. Q. Xie, "Tele-rehabilitation using in-house wearable ankle rehabilitation robot," Assistive Technology, vol. 30, no. 1, pp. 24-33, 2018.

[11] H.-S. Kim and G.-S. Kim, "Design of force sensors for the ankle rehabilitation robot of severe stroke patients," Journal of Sensor Science and Technology, vol. 25, no. 2, pp. 148-154, 2016.

[12] H. G. Cha and M. K. Kim, "Effects of strengthening exercise integrated repetitive transcranial magnetic stimulation on motor function recovery in subacute stroke patients: a randomized controlled trial," Technology and Health Care, vol. 25, no. 3, pp. 521-529, 2017.

[13] M. Zhang, S. Q. Xie, and X. Li, “Adaptive patient-cooperative control of a compliant ankle rehabilitation robot (CARR) with enhanced training safety," IEEE Transactions on Industrial Electronics, vol. 65, no. 2, pp. 1398-1407, 2017.

[14] Y. Ren, Y. N. Wu, and C. Y. Yang, "Developing a wearable ankle rehabilitation robotic device for in-bed acute stroke rehabilitation," IEEE Transactions on Neural Systems and Rehabilitation Engineering, vol. 25, no. 6, pp. 589-596, 2016.

[15] K. P. Michmizos and H. I. Krebs, "Pediatric robotic rehabilitation: current knowledge and future trends in treating children with sensorimotor impairments," NeuroRehabilitation, vol. 41, no. 1, pp. 69-76, 2017.

[16] K. Kim and K. Jeon, "Development of an efficient rehabilitation exercise program for functional recovery in chronic ankle instability," Journal of Physical Therapy Science, vol. 28, no. 5, pp. 1443-1447, 2016.

[17] M. Liu, Y. Jia, and N. Shi, "Application value of an integrated treatment model of orthopedic rehabilitation in patients undergoing total knee arthroplasty," International Journal of Clinical and Experimental Medicine, vol. 11, no. 9, pp. 9455-9461, 2018.

[18] A. J. Sheean, D. J. Tennent, J. G. Owens, J. M. Wilken, J. R. Hsu, and D. J. Stinner, "Effect of custom orthosis and rehabilitation program on outcomes following ankle and subtalar fusions," Foot \& Ankle International, vol. 37, no. 11, pp. 1205-1210, 2016.

[19] S. Hussain, P. K. Jamwal, and M. H. Ghayesh, "State-of-theart robotic devices for ankle rehabilitation: mechanism and control review," Proceedings of the Institution of Mechanical Engineers, Part H: Journal of Engineering in Medicine, vol. 231, no. 12, pp. 1224-1234, 2017.

[20] J. G. Jiang, X. F. Ma, and B. Huo, "Recent advances on lower limb exoskeleton rehabilitation robot," Recent Patents on Engineering, vol. 11, no. 3, pp. 194-207, 2017.

[21] J. L. Chang, R. Y. Lin, M. Saul, P. J. Koch, H. I. Krebs, and B. T. Volpe, "Intensive seated robotic training of the ankle in patients with chronic stroke differentially improves gait," NeuroRehabilitation, vol. 41, no. 1, pp. 61-68, 2017.

[22] H. Jansen, M. Jordan, S. Frey, S. Hölscher-Doht, R. Meffert, and T. Heintel, "Active controlled motion in early rehabilitation improves outcome after ankle fractures: a randomized controlled trial," Clinical Rehabilitation, vol. 32, no. 3, pp. 312-318, 2018.

[23] H. Rakhodaei, M. Saadat, A. Rastegarpanah, and C. Z. Abdullah, "Path planning of the hybrid parallel robot for ankle rehabilitation," Robotica, vol. 34, no. 1, pp. 173-184, 2016.

[24] J. C. Perez-Ibarra, A. A. G. Siqueira, and M. A. Silva-Couto, "Adaptive impedance control applied to robot-aided neurorehabilitation of the ankle," IEEE Robotics and Automation Letters, vol. 4, no. 2, pp. 185-192, 2018.
[25] Y. Charbel, T. Podkopai, and D. Podkopai, "Results of using professional motor test in the program of physical rehabilitation of football players after injuries of the ankle joint," Slobozhanskyi Herald of Science and Sport, vol. 7, no. 2, pp. 15-19, 2019. 